\title{
CLASSIFICATION SCHEME FOR STATISTICAL THEORIES WITH UNUSUAL SYMMETRIES
}

\author{
Francisco C. ALCARAZ \\ Departamento de Fiśica, Universidade Federal de São Carlos, 13560 São Carlos, SP, Brasil
}

Laurence JACOBS

AT\&T Bell Laboratories, Holmdel, NJ 07733, USA

Robert SAVIT*

Physics Department, University of Michigan, Ann Arbor, MI 48109, USA

Received 4 December 1984

\begin{abstract}
A large class of theories with unusual symmetries is constructed and discussed from a unified viewpoint. These are statistical models with symmetries which correspond to an interpolation between standard globally-invariant theories and theories with a local symmetry. The models describe the dynamics of $\mathrm{U}(1)$ (or $\mathrm{Z}(N)$ ) variables, $\mathrm{e}^{i \phi}$, located at the sites of a lattice of arbitrary dimension and geometry. We describe a classification of these theories based on what we call their symmetry index: the value of $\boldsymbol{n}$ for which the hamiltonian of a $d$-dimensional system is invariant under the transformation $\phi\left(x_{1}, \ldots, x_{d}\right) \rightarrow \phi\left(x_{1}, \ldots, x_{d}\right)+\Lambda\left(x_{1}, \ldots, x_{d}\right)$, where $\Lambda$ satisfies a set of $n$ linearly-independent constraints. We discuss how the critical properties and the structure of topological defects of a model are determined by $d$ and $n$.
\end{abstract}

\section{Introduction}

The most usual types of statistical models are those with either a global symmetry (such as the Ising or $x-y$ models), or a local gauge symmetry. However, a number of interesting models recently discussed in the literature [1], among which are some theories thought to describe some of the properties of liquid crystals and helical magnets, have symmetry properties which are intermediate between global and local symmetries. In this paper we discuss a scheme capable of systematically classifying such theories. As we shall discuss below, the groupings of our classification scheme are almost certainly related to universality classes. Furthermore, models which have these unusual symmetries typically have multibody interactions in their hamiltoni-

* A.P. Sloan Foundation Fellow. Research supported in part by the National Science Foundation under grant PH78-0846. 
ans. Thus our classification scheme may be thought of as a systematic procedure for classifying many theories with multibody interactions.

The key to our classification procedure is the introduction of a new quantity, $n$, which we call the symmetry index of the theory. Roughly speaking, $n$ is a measure of the dimension of the subspace over which the gauge function of the theory is constrained. To be more precise, let us consider (as we shall in the bulk of this paper), a $\mathrm{U}(1)$-invariant theory of a single field, $\mathrm{e}^{i \phi}, \phi \in(-\pi, \pi]$, associated with the sites of some hypercubical lattice in $d$ dimensions. Then we can construct theories with a hamiltonian, $H(\phi)$, which is invariant under the transformation

$$
\phi\left(x_{1}, \ldots, x_{d}\right) \rightarrow \phi\left(x_{1}, \ldots, x_{d}\right)+\Lambda\left(x_{1}, \ldots, x_{d}\right) .
$$

If $\Lambda$ satisfies a set of $n$ linearly independent differential constraints, the theory will be said to have a symmetry index equal to $n$. When $n=0(n=d)$ the theory will have a truly local (global) symmetry. (Actually, as we shall see below, there seems to be no simple way of writing an $n=0$ theory with just one field, a fact perhaps not surprising to aficionados of gauge theories.) For $0<n<d$ the theory has a symmetry which is of a new type and is, in a certain sense, intermediate between local and global symmetries.

There are many different kinds of differential constraints which can be imposed upon $\Lambda$. However, the simplest kinds of linear differential constraints are those of the form $\Delta_{i} \Lambda=0$, with $\Delta_{i}$ a first-order differential operator in the $x_{i}$ direction, which force $\Lambda$ to be independent of $x_{i}$. In much of this paper we will deal explicitly with theories whose gauge functions satisfy such constraints, but the majority of our results apply to a much broader class of theories, as we shall discuss below.

In subsect. 2.1 we will present our classification scheme. We shall also introduce the notion of compound symmetries, that is, situations in which $\Lambda$ is constrained (e.g. constant) on different subspaces, $S_{1}, \ldots, S_{j}$ (which may have different dimensions) of a $d$-dimensional space, but is not similarly constrained over the entire subspace spanned by $S_{1}, \ldots, S_{j}$. Because theories of the type we are discussing are rather unusual, it is helpful to have some simple, useful realizations of them. Therefore, in subsect. 2.2 we will construct a hierarchy of theories for all $n$ and $d$. These theories, with gauge functions satisfying constraints of the form $\Delta_{i} \Lambda=0$, will generally be the simplest non-trivial realizations of a model for a given $n$ and $d$. In subsects. 2.3 and 2.4 we will return to our general classification scheme and will present a discussion of the duality properties and topological excitations of theories with $0<n<d$. Sect. 3 includes a brief discussion of a recently proven generalization of the Mermin-Wagner theorem [2] which shows that a U(1)-invariant theory with $n \leqslant 2$ in any dimension has no long-range order for $T>0[3,4]^{\star}$. This result is indicative of the usefulness of our classification procedure: the value of $n$ is clearly a

\footnotetext{
* Several specific examples of $n=2$ three-dimensional theories are discussed in detail in [4].
} 
relevant parameter in determining the critical properties of a theory. Relative to usual globally-symmetric statistical models, it is, in a certain sense, a generalization of the concept of dimension. In this section we also speculate about the kinds of symmetry breaking that may occur for general $n$ and $d$, and describe the extensions of our results to theories whose gauge functions satisfy more complex constraints. This last point is important, since some of the models germane to liquid-crystal systems are of this type. Finally, sect. 4 consists of a brief summary and conclusions.

\section{Index- $n$ symmetries}

\subsection{THE CLASSIFICATION SCHEME}

Consider a U(1)-invariant theory of a single field, $s(\boldsymbol{r})=\mathrm{e}^{i \phi(r)}$, with $\phi \in(-\pi, \pi]$. Suppose, for simplicity, that the theory is defined on a $d$-dimensional simple hypercubic lattice. (This restriction is not necessary, but will make our presentation easier.) We associate the spins with the sites of the lattice. We shall be interested in discussing theories without explicit long-range interactions and with hamiltonians which are invariant under the transformation

$$
\phi\left(x_{1}, \ldots, x_{d}\right) \rightarrow \phi\left(x_{1}, \ldots, x_{d}\right)+\Lambda\left(x_{n+1}, \ldots, x_{d}\right),
$$

in which $\Lambda$ is an arbitrary function of its arguments. Such a theory will be said to have a symmetry index equal to $n$. (In fact, many of our considerations will apply equally well to theories where $\Lambda$ is a function of all $d$ coordinates, but satisfies $n$ conditions of the form $O_{j} \Lambda=0, j=1, \ldots, n$, where the $O_{j}$ are certain linearly-independent differential operators. This will be discussed in more detail below.)

In general we will consider theories whose hamiltonians have the form

$$
H(\phi)=\sum_{x} \sum_{p=1}^{s} f_{p}\left(\sum_{j=1}^{q(p)} c_{p j} \phi_{j}\right),
$$

where the $f_{p}$ are functions of a linear combination of $q(p) \phi$ 's and the $c_{p j}$ are some set of constant coefficients. The coordinate of $\phi_{j}$ is $x+r_{j}$, and since we assume there are no explicit long-range interactions, $\left|\boldsymbol{r}_{j}\right|$ is finite. Although our considerations are really more general, it will be helpful to think of the interactions in (2.2) as being defined on the $k$-dimensional $(k \leqslant d)$ simplices of the $d$-dimensional lattice. For example, nearest-neighbor interactions along links, four-body interactions around a plaquette, etc. We shall discuss the more general case later.

What kinds of symmetries can our theories have? To begin, we note that if a theory has an index- $n(n<d)$ symmetry then, a fortiori, it has an index- $n^{\prime}$ symmetry 
with $d \geqslant n^{\prime}>n$. This lower symmetry is just the symmetry expressed by considering those transformations in which $\Lambda$ is a function only of a subset of the coordinates $x_{n+1}, \ldots, x_{d}$. A theory in which these are the only symmetries and in which there is only one subspace for which (2.1) is satisfied will be said to have a simple index- $n$ symmetry. A theory for which (2.1) is valid for several distinct sets $\left\{x_{n+1}, \ldots, x_{d}\right\}$ will be said to have a multiple index-n symmetry. It is also possible for a theory to have an index- $n$ symmetry (simple or multiple) as well as a distinct index- $m$ symmetry (also simple or multiple) with $m>n$ (i.e. such that $\left\{x_{m+1}, \ldots, x_{d}\right\}$ is not a subset of $\left.\left\{x_{n+1}, \ldots, x_{d}\right\}\right)$ but not an index- $(n+m)$ symmetry. Such a theory will be said to have a compound symmetry.

In addition to symmetries of the form (2.1), it is possible to construct theories with symmetries such that $H(\phi)$ is invariant under the more general transformation (1.1) in which $\Lambda$ is a function of all coordinates, but satisfies a set of $n$ constraints of the form

$$
O_{1} \Lambda=O_{2} \Lambda=\cdots=O_{n} \Lambda=0
$$

where the $O_{j}$ are a set of $n$ linearly-independent difference operators. Most of the discussion of the next two sections will refer explicitly with theories invariant under (2.1), but as will be apparent, most of the main conclusions apply as well to the larger class of theories invariant under (2.3).

\subsection{CONSTRUCTING SIMPLE MODELS WITH INDEX- $n$ SYMMETRY}

We shall now construct a hierarchy of simple theories invariant under (2.1) for all $n$ and $d$. Consider the case of a theory with an index- $n$ symmetry. We consider only theories that are non-trivial in the sense that they are a priori coupled throughout the entire $d$-dimensional space (although we do allow the possibility that they may be trivial in the sense that they may be transformed into a set of finite non-interacting theories). Then, the simplest theory of the form (2.2) which possesses this symmetry has $s=n$. Such a theory can be constructed by having one term in (2.2) in which the spins at the corners of a $(d-n+1)$-dimensional element of the lattice are coupled together, and $n-1$ other terms which are simple two-body, nearestneighbor interactions. The $(d-n+1)$-dimensional interaction is defined on a $(d-n$ $+1)$-dimensional simplex of the lattice whose edges span a specific $(d-n+1)$ dimensional subspace of the $d$-dimensional lattice, and each of the $n-1$ nearestneighbor interactions occurs along one of the $n-1$ directions which is orthogonal to the subspace spanned by the aforementioned simplex. The $c_{p j}$ are chosen to ensure that the symmetry is satisfied. For example, in these simple cases the $c_{p j}$ may be chosen to alternate between +1 and -1 as we trace around the simplex where the interaction is defined. 
A few examples will clarify the situation. First notice that this procedure gives no theory with $n=0$, since for $n=0$ one would require a $(d+1)$-dimensional simplex on a $d$-dimensional lattice. Without including other fields in the problem, we know of no way of constructing such a theory. Turning to the less pathological case of $n>0$, we see that in three dimensions we can have a multiple index-2 symmetry by including in (2.2) a four-body interaction on each plaquette in the $\left(x_{1}, x_{2}\right)$ plane, and a nearest-neighbor two-body interaction in the $x_{3}$ direction. Then $H$ is invariant under (2.1) with $\Lambda=\Lambda\left(x_{i}\right), i=1,2$. To obtain a theory with symmetry index equal to one for $d=3$ we include in $H$ only a single eight-body interaction which involves all the spins which lie at the corners of a given elementary cube of the lattice and then sum over all such cubes. With the proper choice of coefficients, $c_{1 j}$ in (2.2), it is clear that this theory will then have a three-fold index-1 symmetry, so that the hamiltonian is invariant under (2.1) with $\Lambda=\Lambda\left(x_{i}, x_{j}\right), i, j=1,2,3$. (This theory is actually trivial in the sense that it can be decoupled into a set of non-interacting trivial theories. The reason for this can be traced to the fact that the theory has index-1 symmetries. This point is discussed in more detail elsewhere [4].) A little thought reveals that, in general, a theory designed with the above construction will have a $(d-n+1)$-fold index- $n$ symmetry. (This statement becomes more obvious if this hierarchy of theories is considered in continuum notation in which case the argument of a $k$-dimensional interaction becomes $\partial_{1} \partial_{2} \cdots \partial_{k} \phi$, where $\partial_{j} \equiv \partial / \partial x_{j}$.) It is of course possible to construct more complicated theories with similar symmetry properties or with different multiples of the index- $n$ symmetry. But the procedure outlined above produces, in a certain sense, the simplest such theories. We will have occasion here and elsewhere [4] to discuss in detail a few more complicated theories, but for now we turn to an exploration both of some of the general features of systems with an index- $n$ symmetry, and also of some of the systematics of the hierarchy of theories we have just constructed.

\subsection{DUALITY PROPERTIES}

Let us first describe the duality properties of theories included in our general classification scheme. This is most easily done by referring to the following general result [5]: an abelian theory of a single scalar field, the hamiltonian of which can be written as a sum of $k$ distinct terms, has a dual representation which can be written as a statistical theory of $k-1$ independent fields. Furthermore, these $k-1$ independent fields can be augmented with a set of dependent gauge fields so that the dual form of the theory has a generalized gauge symmetry. After including the extra gauge fields, the hamiltonian can be written as a function of an antisymmetric tensor field, $A_{\mu_{1}, \mu_{2}, \ldots, \mu_{k-2}}$, and the dual hamiltonian will be invariant under a gauge transformation of the general form

$$
A_{\mu_{1}, \ldots, \mu_{k-2}} \rightarrow A_{\mu_{1}, \ldots, \mu_{k-2}}+\Xi\left\{\tilde{O}_{\mu_{1}} \tilde{\Omega}_{\mu_{2}, \ldots, \mu_{k-2}}\right\},
$$


where $\tilde{\Omega}$ is a gauge function with $k-3$ indices, the $\tilde{O}_{\mu}$ are a set of $k$ operators determined by the form of the original hamiltonian, and $\Xi\{\}$ denotes an antisymmetrized sum over all permutations of $\mu_{1}, \ldots, \mu_{k-2}$. (For details of the solution, and a method for determining the $\tilde{O}_{\mu}$, see ref. [5].)

Some features of duality transformations that are familiar from more common theories apply here as well: if the group of the original theory is $\mathrm{Z}_{N}$, then the group of the dual theory will be $Z_{N}$ also. If the original theory has a $U(1)$ symmetry, then the dual theory has a $Z_{\infty}$ symmetry. If the hamiltonian of the original theory is only quadratic (or of a Villain-like form), then so will be the hamiltonian of the dual theory. Finally, the coupling constants of the dual theory will be related to those of the original theory in a manner dictated by the symmetry group, just as is the case with more standard (e.g., globally symmetric) theories. On the other hand, one genuine complication that may arise in these more unusual theories is that the natural form of the dual lattice may be rather complicated. This is because the structure of the dual lattice is naturally determined by the geometry associated with the interactions of the original theory rather than simply by the locations of the spins themselves, and since the theories under discussion involve multibody interactions, these geometries can be, in principle, quite involved.

To illuminate the preceding general discussion, let us look at a few simple examples. One of the most interesting classes of theories in our scheme is the index-2 class. Fortunately, the simple index-2 model in $d$ dimensions given by the construction of subsect. 2.2 is self-dual in any dimension, in the sense that the dual theory contains two terms with arguments which have the same functional form as the original hamiltonian. The procedure for calculating the dual form is an exact parallel of that for the $d=2 \mathrm{Z}_{N}$ clock or $\mathrm{U}(1) x-y$ models. The index-3 models in $d$ dimensions generated by the construction of sect. 2.2 are dual to a gauge theory of a single gauge field, $A_{\mu}, \mu=1,2,3$. Note that although the theory and its dual live in $d$ dimensions, the gauge field has only three components. Depending on the form of the interactions in the original theory, these components may or may not be simply associated with spatial directions. Of course, the simplest example of these theories are just the familiar three-dimensional globally-symmetric models, such as the $d=3$ Ising, $\mathrm{Z}_{N}$, or $x-y$ models, in which the indices of the gauge field can be associated with spatial directions.

As a final example, we refer to a theory which is discussed in greater detail in ref. [4]. This is a theory in the index-2 class on a simple cubic lattice in three dimensions. The spins in this theory interact via four-body interactions which are defined by multiplying together the four spins that lie at the corners of a given elementary plaquette of the lattice. (To maintain a simple global symmetry one may define a convention which complex conjugates two of the spins in each interaction.) The hamiltonian is defined by summing this interaction over all the plaquettes of the lattice. The dual to this theory is again a gauge theory of a single gauge field $A_{\mu}$, $\mu=1,2,3$, however, the natural lattice for the dual theory is somewhat complicated. 


\subsection{TOPOLOGICAL EXCITATIONS}

Given an abelian symmetry, we may separate the ensuing "topological excitations" into two categories. If the symmetry is discrete (e.g. $\left.Z_{N}\right)$, then there will be some sort of generalized domain-wall excitations. For example, the $d=3 \mathrm{Z}_{N}$-symmetric clock model has orientable domain walls separating regions of spin of different orientation. In addition, there may be other excitations associated not with the discreteness of the symmetry group, but rather with its compactness. For example, the vortex strings that appear in the $\mathrm{U}(1)$-invariant three-dimensional $x-y$ model are of this type.

In this subsection we will sketch some general features of this latter type of excitation. We expect to find these kinds of excitations in $\mathrm{Z}_{N}(N>3)[6]$ and (more simply) in $\mathrm{U}(1)$-symmetric theories. In addition, there may be domain-wall-like excitations if the symmetry group is discrete. These will usually have non-trivial interactions with the topological excitations generated by the compactness of the group. This additional complication, which can greatly enhance the richness of the phase structure, should be borne in mind when passing from a U(1)- to a $Z_{N}$-symmetric theory. (For a discussion of such effects in the $d=2$ clock models see ref. [7].)

Consider a theory with an index- $n \mathrm{U}(1)$ symmetry. As pointed out in ref. [5], the nature of the topological excitations is related to the form of the dual theory. The form of the dual theory depends on the number of distinct terms in the original hamiltonian. If the original theory is a theory of a single $\mathrm{U}(1)$ spin and has $k$ distinct terms in the hamiltonian, then we will be able to write the theory in terms of interacting topological excitations labeled by $k-2$ indices. In the simplest cases these indices will be associated with spatial directions, so that the topological excitations will be interpretable as $(k-2)$-dimensional objects. For example, the $d=2 x-y$ model can be written in terms of interacting point vortices, while the $d=3 x-y$ model can be written in terms of interacting vortex strings.

Since the nature of the topological excitations depends on the number of distinct terms in the original hamiltonian, the topological excitations are not determined solely by the symmetry index, nor by the spatial dimension. We can say, however, that given a (non-trivial) theory with an index- $n$ symmetry, its topological excitations must be at least $(n-2)$-dimensional objects. As we pointed out in subsect. 2.2, the simplest theory with an index- $n$ symmetry has $n$ terms in its hamiltonian, and these theories will have $(n-2)$-dimensional topological excitations. So, for example, the simple index-2 theories in $d$ dimensions will have point vortices embedded in a $d$-dimensional space. On the other hand, the index-2 theory described in the last subsection, and discussed more fully in ref. [4], whose hamiltonian is a sum over all four-body plaquette interactions has topological excitations which are one-dimensional, string-like objects.

In the usual familiar theories such as the two- and three-dimensional $x-y$ models, there are conservation constraints on the topological excitations. In two 
dimensions the constraint may (depending on the boundary conditions) take the form of an overall charge neutrality condition which states that the sum of vortex charges must vanish. In three dimensions, the conservation constraint forces the divergence of the vortex-string current to vanish (except possibly, depending on boundary conditions, at infinity). That is, it enforces $\nabla_{\mu} J_{\mu}=0$, where $J_{\mu}$ is an integer-valued current representing the vortex string and $\nabla_{\mu}$ is a finite-difference operator [8]. In the more general cases of interest in this paper, there will again be constraints associated with the topological excitations, but they may not take quite as simple a form as those discussed above. A review of the derivation leading to the constraint on the divergence of the string current for the $d=3 x-y$ model shows [8] that the fact that the operators that appear in this linear constraint are the $\nabla_{\mu}$ is intimately related to the fact that the operators that appear in the original hamiltonian are also $\nabla_{\mu}$. In general cases where the operators defining the interactions in the original hamiltonian are other than simple first-difference operators, there will be other operators appearing in the constraints on the topological excitations. For example, if the original $\mathrm{U}(1)$ hamiltonian is of the form (2.2) with $s=3$, then the string-like topological excitations, $J_{\mu}, \mu=1,2,3$, will satisfy a constraint of the form $D_{\mu} J_{\mu}=0$, where the $D_{\mu}$ are certain finite-difference operators, each one of which (when operating on the field $\phi_{j}$ ) produces the linear combination of $\phi$ 's which is one of the arguments of one of the terms in (2.2). (Note that, in general, $\mu$ does not refer to a space direction but is merely a label related to the index $p$ in (2.2).)

The formulation of topological excitations we have presented above is quite elegant and useful, a statement which becomes apparent after working through several examples (see, e.g. ref. [4]). Nevertheless, it may not be the most appropriate formulation of the problem in some specific cases. Since the topological currents which satisfy the conservation laws like (for the case $s=3$ ) $D_{\mu} J_{\mu}=0$ can themselves be written in terms of another set of fields (e.g., in the $d=3 x-y$ model [8], $J_{\mu}=\varepsilon_{\mu \nu \lambda} \nabla_{\nu} B_{\lambda}$ ) it may be advantageous for certain purposes to consider the theory as a function of a set of variables other than the $J_{\mu}$ 's. This is particularly so in cases such as the ones considered in this paper for which the operators appearing in the hamiltonian are more complicated than simple first-order finite-difference (or differential) operators. In such cases it is often difficult to provide a simple interpretation of the topological excitations in terms of the original degrees of freedom of the theory. This problem does not occur in the simple $x-y$ model. In that theory, the hamiltonian only includes nearest-neighbor terms, so it is quite clear that a vortex just represents the integer-valued part of an angle change as we move from site to nearest-neighbor site around a closed path in the lattice. With more complicated interactions such an interpretation is not always possible, and, depending on the theory, a close examination may reveal a more intuitively appealing set of variables which are functions of the $J_{\mu}$ 's. Such a situation takes place in the model studied by Amit, et al. [9]. In that model the mathematically natural set of topological 
excitations are point-like vortices in three dimensions, but it is likely that the physically natural set of topological excitations are string-like variables.

\section{Remarks on the phase structure}

Because the theories we are considering here have a very rich symmetry structure, a number of possibilities for symmetry breaking and phase transitions suggest themselves. Consider, for example, a theory in four dimensions with an $n=3$ symmetry, and for definiteness let us focus on the $n=3, d=4$ theory constructed according to the prescription in subsect. 2.2. Let $(i, j, k, l)$ denote the four spatial directions on the four-dimensional hypercubic lattice with a two-body, nearestneighbor interaction along the $l$-direction. We may define a number of different correlation functions which can test a number of different possible types of symmetry breaking. For example, it may be possible to break the $n=3$ symmetry without breaking the global symmetry. That is, we can imagine a phase of the system in which the correlation functions

$$
C_{m}=\langle\cos [\phi(x)-\phi(x+\hat{m})]\rangle, \quad m=i, j, k,
$$

have non-zero values, while the global spin expectation value, $\left\langle\mathrm{e}^{i \phi}\right\rangle$, is zero. (Of course, the opposite situation cannot occur.) We can therefore imagine a scenario in which there is no symmetry breaking at high temperature, but as we lower the temperature the system proceeds through a sequence of phase transitions leading to phases of increasingly greater broken symmetry. On the other hand, it is possible that all symmetry breaking occurs at the same temperature, so that we go from a phase of unbroken symmetry to a phase in which the symmetry, even the global symmetry, is completely broken. It is not clear which of these options (or other possibilities) prevails. But, in any case, we will be confronted with a new type of phase transition, and so the problem is well worth pursuing.

In the special case of an $n=2$ (or $n=1$ ) symmetry more is known. It has been shown [3] in this case that there is no symmetry breaking in a U(1)-invariant system for $T>0$ in any dimension. The proof is a generalization of that of the MerminWagner theorem [2] and applies both to the global as well as the full semi-local symmetries that the theory possesses. While the proof was presented for the class of $n=2$ theories invariant under (2.1), it is straightforward to see that it also applies to a much larger set of theories invariant under (1.1) subject to the constraints (2.3). This result is a strong indication of the relevance of the symmetry index in determining the critical properties of our systems, and suggests that, just as $d=2$ is the lower critical dimension for ordinary globally-symmetric statistical theories, $n=2$ is the lower critical symmetry index for the more general class of theories discussed here. 
Finally, we remark that it would be interesting to study the phase properties of theories with $0<n<d$ which have discrete or non-abelian symmetries. The latter set of theories is particularly intriguing, since it is not clear how to systematically generalize our class of theories (or even the simple hierarchy constructed in subsect. 2.2) to the non-abelian case.

\section{Summary and conclusions}

In this article we have introduced, in a systematic way, a new class of statistical theories whose symmetries are neither global nor local, but are something intermediate between the two. These theories are characterized by a symmetry index, $n$ which represents the number of constraints which must be satisfied by the gauge function.

After defining these theories in general terms, we described a procedure for constructing a hierarchy of relatively simple, easy to study models with various values of $n$ and $d$. Next we returned to the general classification scheme of subsect. 2.1 and discussed some of the most important and universal features of duality transformations and topological excitations realized by the theories included in this scheme. We then briefly discussed a theorem [3] which shows that, for $n \leqslant 2$, in any dimension, $\mathrm{U}(1)$-invariant theories do not have long-range order. This theorem implies that it is not the dimensionality of the system which is involved in determining this feature of its critical behavior, but rather, what is relevant is the dimensionality of the space over which the symmetry is constrained or effectively global. In this sense, the symmetry index represents a generalization of the notion of spatial dimension.

Our results represent the beginning of a classification scheme for a new set of theories which are statistically rich and which may be relevant in the description of important physical systems [1]. In addition, it is, to our knowledge, the first scheme capable of systematically classifying and describing the phase structure of statistical theories with multi-body interactions.

A number of aspects of these theories have not yet been addressed in detail. For example, it is almost certain that our classification scheme is related to universality classes. Therefore, a more complete analysis of the critical properties of these systems is called for. A knowledge of the general phase structure and critical exponents of the models, which could be obtained, for example, by a systematic finite-size scaling analysis is needed. Similarly, an analysis of the correlation functions in the soft, low-temperature phase is lacking. We hope to be able to address these points in the near future.

We are happy to acknowledge the hospitality of the Institute for Theoretical Physics, Santa Barbara, where this work was begun. The research was supported in part by NSF grant PHY77-27084. 


\section{References}

[1] D. Amit, S. Elitzur, E. Rabinovici and R. Savit, Nucl. Phys. B210[FS6] (1982) 69;

F.C. Alcaraz, L. Jacobs and R. Savit, J. Phys. A16 (1982) 175;

G. Grinstein, J. Phys. A13 (1980) L201; Phys. Rev. B23 (1981) 4615;

L. Jacobs and R. Savit, Ann. New York Acad. Sci. 410 (1983) 281

[2] N.D. Mermin and H. Wagner, Phys. Rev. Lett. 17 (1966) 1133;

F. Wegner, Z. Phys. 206 (1967) 465;

N.D. Mermin, Phys. Rev. 176 (1968) 250;

S. Coleman, Comm. Math. Phys. 31 (1973) 259

[3] F.C. Alcaraz, L. Jacobs and R. Savit, Phys. Rev. Lett. 51 (1983) 435

[4] F.C. Alcaraz, L. Jacobs and R. Savit, to appear

[5] R. Savit, Nucl. Phys. B200[FS4] (1982) 233

[6] M. Einhorn, E. Rabinovici and R. Savit, Nucl. Phys. B170[FS1] (1980) 16

[7] S. Elitzur, R. Pearson and J. Shigemitsu, Phys. Rev. D19 (1979) 3698;

A. Ukawa, P. Windey and A. Guth, Phys. Rev. D21 (1980) 1013;

E. Fradkin and L. Kadanoff, Nucl. Phys. B170[FS1] (1980) 1;

M. Einhorn, E. Rabinovici and R. Savit, ref. [6];

L. Kadanoff, J. Phys. Al1 (1978) 1399

[8] R. Savit, Phys. Rev. B17 (1978) 1340

[9] D. Amit, S. Elitzur, E. Rabinovici and R. Savit, ref. [1] 